\title{
Data Reduction Induced Errors in the Asteroseismology of Early-Type Stars
}

\section{Petr Škoda}

Astronomical Institute of the Academy of Sciences, Czech Republic email: skoda@sunstel.asu.cas.cz

In the asteroseismology of early type stars it plays an important role the extremely precise high resolution spectroscopy. Especially the determination of pulsation modes from observations requires the identification of subtle changes in line profiles of a number of chemical elements. As commonly available instruments suitable for this purpose are echelle spectrographs, there is a number of possible imperfections and even systematic errors introduced by the echelle reduction procedures. We will mention several, most critical steps, that should be carefully checked during every reduction.

The errors in wavelength calibration may be caused by behaviour of two-dimensional dispersion polynomials at the edges of the chip. Further improvements may be achieved by elimination of blended lines and careful line centering algorithms (de Cuyper and Hensberge 1998) or by introduction of a new fitting formula ( Hensberge and Verschueren 1989).

One of the problematic issues is the procedure of cosmics rejection by the variance-weighted optimal extraction based on estimated cross-order profile (Horne 1986). As was shown by Verschueren et al. (1997), it cannot bring measurable improvement on data with high SNR, but can introduce strange artifacts. Therefore the better method is the removal of cosmics from the original 2D frame using statistic medians (Pych 2004) or Laplace transform (van Dokkum 2001). The alternative, self-consistent method of optimal extraction was also suggested ( Piskunov \& Valenti (2002)).

The critical step before merging of separate spectral orders into one long spectrum is the removal of echelle blaze function. It is a very difficult task, some ideas being given by Škoda \& Hensberge (2003), Hensberge (2007) or Škoda et al. (2008). The problem is much worse in case of early type stars, where some $\mathrm{H}$ and He lines may be extended over more that one echelle order, and so the precise order merging is required to preserve the line profile.

\section{References}

de Cuyper, J.-P. \& Hensberge, H. 1998, A\& AS, 128, 409

Hensberge, H. \& Verschueren, W. 1989, The Messenger, 58, 51

Hensberge, H. 2007, in The Future of Photometric, Spectrophotometric and Polarimetric Standardization, ASP Conf. Series, 364, 275

Horne, K. 1986, PASP, 98, 609

Piskunov, N. E. \& Valenti, J. A. 2002, A\&A, 385, 1095

Pych, W. 2004, PASP, 116, 148

Škoda, P. \& Hensberge, H. 2003, in Astronomical Data Analysis Software and Systems XII, ASP Conf. Series, 295, 415

Škoda, P., Šurlan B \& Tomić, S. 2008, Proc. SPIE, 7014, 196

van Dokkum, P. G. 2001, PASP, 113, 1420

Verschueren, W., Brown, A. G. A., Hensberge, H., David, M., Le Poole, R. S., de Geus, E. J., \& de Zeeuw, P. T. 1997, PASP, 109, 868 